\title{
Using Machine Learning Methods in the Financial Market for Technical Analysis Based on Hybrid Models
}

\author{
Amir Hossein Sabbaghi Lalimi and Hamed Damavandi*
}

Industry Engineering, Department of Socio-Economic Systems, Sharif University of Technology, Iran.

\section{*Corresponding Author: \\ $>$ damavandi@gmail.com}

Received: 15 September, 2020

Accepted: 30 October, 2020

Published: 25 November, 2020

\begin{abstract}
In this study, it is presented a new hybrid model based on deep neural networks to predict the direction and magnitude of the Forex market movement in the short term. The overall model presented is based on the scalping strategy and is provided for high frequency transactions. The proposed hybrid model is based on a combination of three models based on deep neural networks. The first model is a deep neural network with a multi-input structure consisting of a combination of Long Short Term Memory layers. The second model is a deep neural network with a multi-input structure made of a combination of one-dimensional Convolutional Neural network layers. The third model has a simpler structure and is a multi-input model of the Multi-Layer Perceptron layers. The overall model was also a model based on the majority vote of three top models. This study showed that models based on Long Short-Term Memory layers provided better results than the other models and even hybrid models with more than $70 \%$ accurate.
\end{abstract}

Keywords: Forex prediction, Machine learning, Deep learning, High frequency trading, Neural network, Long short-term memory convolutional neural network, Multi-Layer perceptron

\section{Introduction}

Financial markets have always been an attractive option for investors seeking higher returns than bank profits. different methods have been used to predict the market and make decisions due to the great attention that different investors have paid to this market, over time. old methods such as linear models lost their efficiency and accuracy over time due to the large number of financial market players and the resulting complexities. Therefore, it is needed to use models that can model this complexity and nonlinear relationships well.

Today, the use of data-driven models in this market has been considered by the investors and researchers, and by using these models, they have been able to study the various dimensions of this market and its variables to the desired extent. Machine learning techniques are some of these methods such as deep artificial neural networks that have recently been considered in financial markets. These methods have been able to perform better than the other methods due to the large number of influential variables in the market. in some cases, older statistical models in recent years could not cover their various dimensions and complexity due to the special characteristics of financial data. As a result, the use of data-based models, especially deep neural networks, has proved its place in forecasting and decision-making.

Therefore, there is a need to introduce new models of machine learning in order to increase the accuracy of forecasting, or improve decision-making in the market. To solve this problem, some hybrid models were proposed to cover the weaknesses of each model individually and improve their overall performance. For this purpose, some machine learning techniques such as various types of artificial neural networks, time series models or the other models can be combined with each other.

The purpose of this paper is to present a hybrid network model to improve the accuracy of forecasting the direction and size of movement of the euro-dollar pair in the Forex market. We will see that these models 
are a part of machine learning models to increase the trading power of the trader in a great extent.

\section{Literature review}

Fortunately, in today's data age, this can be bypassed, as a new train with the idea of "let the data speak for itself" has been proposed and is receiving more attention. Contrary to information from newspapers, microblogging and Twitter, the daily trading data that takes place in business systems is quite realistic. The rapid development of machine learning provides many new opportunities to use this trading data to predict stock price movement. In fact, the use of machine learning to predict stocks has been studied for over thirty years.

Early studies in the 1990s focused mainly on the use of neural networks for forecasting [1], which to some extent refuted the validity of the efficient market hypothesis [2]. For example, Tsiboris and Seidenberg [3] used neural networks to predict stock prices based solely on past stock prices. The performance of these early methods was usually not good due to the limited size of neural networks. To address this issue, some recent studies have resorted to a combination of models [4], [5], or a combination of models [6], [7], and [4]. All of the above studies have one common weakness that their practical availability is still questionable. In their studies, small amounts of data were carefully selected and labeled and used to training and test patterns. Since the data does not cover all stocks and their movements on the stock exchange, the generalization capabilities of the models in real applications are reduced.

The financial market trades heavily every day. We cannot expect a real-world computer-based decisionmaking system that relies heavily on humans to select and label the data used to training the model. Recognizing the unsupervised pattern is becoming more important in today's big data age [8]. If the problem of automated data processing is not solved, even if the learning algorithms used are advanced, the system can hardly be led to a real use.

After using different prediction models, the researchers attempted to combine different models to improve the accuracy of the prediction and cover the weakness of each one. Tsai and Wang [9] combined the decision tree and the neural network to make a forecast of Taiwan stock market; the accuracy of their hybrid model is about $70 \%$, however, their test data set was relatively small, containing only dozens of stocks.

Reader [10] also introduced another hybrid model for stock price forecasting that combines linear and nonlinear algorithms. He used two time series, ES (Exponential Smoothing), ARMA (Auto regressive moving average) and the RNN (Recurrent neural network). He used ARMR time series parameters to input its neural network. He then weighed each of three predictors and optimized it with a genetic algorithm.

\section{Problem definition}

Our problem is to provide a machine learning model to predict the movement of the Forex market in the short term and ultimately provide a trading strategy. In the Forex market, several currency pairs such as Euro / Dollar, Pound / Dollar, Dollar / Yen, Franc / Dollar, etc. are exchanged. We designed our model for the Euro / Dollar pair so that we can predict the direction of the pair in the short term.

Since the Forex market is a two-way market, the trader can make a profit both in the descent and fall of the market, which adds the attractiveness to this market. The trader can also trade in this market both long-term and short-term. In short trades, which can be as short as a second, the trader makes a higher number of trades in a certain period of time. Short-term strategy seems to be more profitable than long-term strategy when it is accompanied by proper accuracy.

Traders mainly use two types of technical or fundamental analysis to improve the forecast for market movement. In Forex technical analysis, like the other financial markets, we encounter various indices and lines that are effective in predicting the direction and intensity of the market trend. Also, in the fundamental analysis of the Forex market, attention is paid to economic and political news and reports. The proposed model of this research is presented to predict the movement of Euro / Dollar pair ratio in the short term, in which technical indices are used.

Among the various models that can be offered to predict the market, we chose the hybrid model, which is generally more accurate. In the proposed hybrid model, three different machine learning models are used, which are combined by majority voting method. In the majority voting method, the prediction of general model corresponds to the prediction that has the votes of majority of the models.

\section{Scalping strategy}

To design a forecasting model, we first looked at the different trading strategies used by traders so that we could get ideas from them to build a new model. Among them, we focused on short-term scalping strategies. Finally, we chose a scalping strategy and built our model based on it.

Scalping is a trading method that focuses on making a profit from minor changes in market trends. This strategy requires a trader who has a difficult exit strategy, because a large loss can waste several small profits that the trader has already made. This strategy requires tools such as live access to the market and 
direct access to the broker, and the trader must be able to record a large number of orders in the short term [11].

Scalping is based on the premise that most market trends complete the first step of a move. But it is unclear where they will go next. After the first stage, sometimes the trends continue to move and sometimes they stop moving [11].

A scalpel increases its number and not the amount of its small profits. This is the opposite of the view that glorifies: "Let profit continue". Scalping strategy gets good results when a person increases the number of wins in his strategy and sacrifices the amount of his profit. A successful scalpel has a high win-loss ratio in its strategy and also considers its profit equal to or slightly more than its loss [11].

The scalping strategy which we designed our model based on that, is the strategy of colliding two moving average prices. This strategy is based on the collision of two short-term and long-term moving averages. To examine the collision of two moving averages, we consider two short-term and long-term time intervals. The short-term interval is the trading interval, and the long-term interval helps us to more accurately predict the direction of movement in the short term.

In order to implement this strategy, we considered the long-term time interval to be one hour and the shortterm time interval to be five minutes, which is our trading interval. In the collision of two moving average prices, we should know that when the short-term moving average crosses the long-term moving average, it will be a signal to climb the trend. Conversely, if the short-term average breaks the long-term average, we will have a signal for the market to decline. For example, if the short-term average breaks the long-term average downwards over a period of five minutes, we will probably face a downtrend. But using the collision of two moving averages in only one interval as our only signal, will cause us many errors. Therefore, we need to look for another signal as our trading strategy. We use the long-term interval as confirmation for the shortterm signals.

In the long-term interval, if the short-term moving average breaks the long-term moving average, it indicates that the long-term trend is likely to be hourly. Now, if at the same time, in the short term, the longterm average breaks downwards, since the long-term trend is also downtrend, the downtrend signal will be stronger. But if, on the contrary, it breaks another short-term average upwards, the uptrend signal will not be very strong, considering that the overall downtrend will not be strong. We can also use technical indices to increase the accuracy of the received signals. The technical indices used in the model are RSI, Williams $\%$ R, ADX, PDI, MDI, EMA.

Given the above, we focused on points that are in the trading interval (short interval of five minutes) exactly after the collision of two moving averages. The moving averages are exponential and for both five minute and one hour time intervals, we considered the short-term average to be three periods and the long-term average to be fourteen periods. We also calculated moving averages for closing prices. Post-collision points can be divided into the following four groups:

The first group are the points that just before that, in a period of five minutes, the moving average of three periods cut the average of fourteen periods upwards. Also, in these points, in a period of one hour, the moving average of three periods is above the average of fourteen periods. In this case, we will see a strong bullish signal.

The second group are the points where, just before them, in a period of five minutes, the moving average of three periods cut the average of fourteen periods downwards. Also, in these points, in a period of one hour, the moving average of three periods is above the average of fourteen periods. In this case, we will see a weak bullish signal.

The third group are the points that just before that, in a period of 5 minutes, the moving average of three periods cut the average of fourteen periods downwards. Also, in these points, the moving average of three periods is below the average of fourteen periods in a period of one hour. In this case, we will see a strong bearish signal.

The fourth group are the points that just before that, in a period of five minutes, the moving average of three periods cut the average of fourteen periods down. Also, in these points, in a period of one hour, the moving average of 3 periods is above the average of fourteen periods. In this case, we will see a weak bearish signal.

Among the above four groups, we selected the first and third groups that have stronger ascent and descent signals. Now we have to predict the points that have relatively stronger signals, after which the market will move up or down enough. Because in the Forex market, the buying and selling price of a currency pair is different and the buying price is always higher than the selling price. This causes any transaction to be costly for the trader, and if the trend does not descent or fall enough, it will cause the trader's loss. The purpose of the model is to predict the points between the first and third groups, after which the market trend will grow or decline enough. The transaction cost per transaction will usually be between ten and fifteen points per Euro / Dollar. For example, if the Euro / Dollar pair ratio is 1.0850 when selling, the same ratio will be 1.0851 to 1.08515 for buying. Therefore, we should look for points where the rate of ascent or descent is more than fifteen points, which is more likely among the points of groups one and three.

Therefore, among the points of the first group, we are looking to predict the points after which we see an increase of more than fifteen points in the value of the 
currency pair ratio. Also, in the third group, we are looking for points after which we see a decrease of more than fifteen points in the value of the currency pair ratio.

\section{Prediction model}

\section{Model output}

The forecast model for the points of the first group determines that after which points, we climb more than fifteen points. In other words, for this group of points, it is determined how much the positive changes in the ratio of the currency pair will be until the next collision of the five-minute moving averages, which will be downward.

The model for the points of the third group also specifies that after which one of them we will have a decent more than 15 points. In other words, it is determined for this group of points how much the negative changes of the currency pair will be until the next collision of the 5-minute moving averages, which will be upward.

In the case of first group, if at a point, the rate of descent of the currency pair ratio after that until the next collision of the averages is more than fifteen points, the label of that point will be one and otherwise zero.

Also, in the case of third group, if the rate of decline of the currency pair ratio after that until the next collision of the averages is more than fifteen points, the label will be point one, otherwise it will be zero.

Therefore, the output of model for both groups will be one or zero, which indicates whether the exchange rate ratio is more than fifteen points or not. In the case of first group, if the number of predicted changes is more than fifteen points, we will enter the purchase transaction. In the case of third group, if the number of predicted changes is more than fifteen points, we will enter into a pair sale transaction.

Depending on the type of output, we have a binary classification problem that only two types of labels should be assigned to each of the inputs. Therefore, the problem of machine learning will be in the form of learning with the observer, where the error rate can be measured and specified for each prediction.

Since the prediction model predicts two different directions for two groups and also the conditions are completely different for two groups, we preferred that a machine learning model be learned and tested separately on two categories. In fact, the same model is made for both categories, which has a different learning and testing process.

\section{Model inputs}

Since the selected scalping strategy predicts the direction of movement using two-time intervals of five minutes and one hour, therefore the model inputs will be in both short-term and long-term groups. Therefore, we will have a model with two input groups, which is different from the general single-input models.

\section{Long-Term inputs}

1. Exponential moving average difference of closing price:

The exponential moving average is a moving average that gives more weight and importance to the newest points. The exponential moving average is also known as the exponential weight moving average. An exponential moving average is significantly more responsive to recent price changes, but a simple moving average applies the same weight to all observations. The relationships between two types of moving averages are as follows (86).

$$
\begin{aligned}
& E M A_{t}=\frac{2}{1+\text { Days }} * \text { Value }_{t}+\left(1-\frac{2}{1+\text { Days }}\right) * E M A_{t-1} \\
& S M A_{t}=\frac{1}{n} * \text { Value }_{t}+\frac{1}{n} * V \text { alue }_{t-1}+\ldots+\frac{1}{n} * V \text { alue }_{t-(n-1)}
\end{aligned}
$$

Relations 1 and 2

Let us now examine the collision of two moving averages and its meaning. For example, when the moving average of three periods breaks the average of fourteen periods upwards, according to the above relations, we conclude that the current value of the variable is greater than its previous values. Such a result can be interpreted as saying that we have an increasing amount of variable and a signal to buy. Conversely, if the average of three periods breaks the average of fourteen periods downwards, we will have a signal to sell. We can use their difference to check the status of two exponential moving averages. The only long-term inputs of the predictive model are the difference between the exponential moving average of three and the average of fourteen periods over an hour period. We can summarize the above statements in such a way that if the said difference is positive, the long-term trend is upward and if it is negative, the long-term trend is downward.

$$
\text { longtermInput } \text { ln }_{h}=E M A_{3 h}-E M A_{14 h}
$$

\section{Relation 3}

\section{Short-Term inputs}

We also have different inputs in the short period of five minutes in which transactions take place. We considered short-term inputs for five candlesticks of the previous five minutes (From the first candlestick after collision of moving averages forward) 1. Exponential Moving Average Price of Closing Price

shorttermInput $1=E M A_{3}-E M A_{14}$

Relation 4 
Here is the moving average of three and fourteen periods for five-minute candlesticks. As above, the positive value of this variable is a signal to increase the ratio of the currency pair and the negative value is a signal to decrease the ratio, but short-term signals are good signals when, as we have said, they are in line with the one-hour long-term signal.

2. Relative Strength Index (RSI):

After examining the directionality of the difference between long-term and short-term moving averages and determining the points with the potential of increasing or decreasing trend, we seek to identify stronger short-term trends. One of the indices that can help us predict the strength of the trend is the relative resistance index. The Relative Strength Index (RSI) is a moving index that measures the magnitude of recent price changes to assess over-selling conditions or overtaking the price of a stock or other asset. The RSI is displayed as an oscillator (a line chart that moves between two extreme levels) and has readings from 0 to 100 (87).

The traditional interpretation of the RSI is that values of 70 or higher indicate that the purchase of an asset is excessive and that we will soon see a sell-off. Also, values below 30 indicate the overactivity of sellers and we will soon see an increase in buyer activity and an increase in prices. The relative strength index can be calculated as follows (87).

$$
R S I=100-\left[\frac{100}{1+\frac{\text { averageGain }}{\text { averageLoss }}}\right]
$$

\section{Relation 5}

In the proposed forecasting model, the three-period relative strength index for five-minute candlesticks is used as one of the short-term inputs to predict the strength of the short-term trend. Since we intended to forecast the market in the short term, we also considered the time period related to the relative resistance index to be short-term.

3. Average Directional Index (ADX):

The average directional index $(\mathrm{ADX})$ is a technical analysis index used by some traders to determine the strength of a trend. This trend can be up or down, which is indicated by two accompanying indices, one is the minus directional index (-DI) and the other is the positive directional index $(+\mathrm{DI})$. Therefore, the ADX usually consists of three separate lines that predict both the direction and strength of the trend. When + DI is higher than -DI, the price goes up, and when -DI is higher than + DI, the price goes down. Also, if the ADX is above 25, we will see a strong trend and if it is below 20, we will have a weak trend. We have refrained from relating to these three indicators due to their long calculations and availability (88). Other short-term inputs of the model are the average directional index and the three-period positive and negative orientational index for five-minute candlesticks. These three indices can help us to determine the direction of the trend and its strength and increase the accuracy of the model. 4. Williams Percent Range Index (Williams\% R Index): Williams percentage range is a moving index that ranges between zero and -100 . Values above -20 means over-buying and the price is approaching its maximum value. Values below -80 also mean over-selling and approaching the price to its lowest level. The relationships related to this index are as follows (89).

Wilians $\% R=\frac{\text { HighestHigh }- \text { Close }}{\text { HighestHigh }- \text { LowestLow }} *(-100)$

Relation 6

Highest High: The highest price of candlesticks in the considered range

Lowest Low: The lowest price of candlesticks in the considered time period

Close: The final price of the last candle

Another short-term input for the Williams Percentage Index is a three-period model for five-minute candlesticks. This index can also be effective in predicting the strength of the trend.

5. Opening, Closing, Highest and Lowest Prices:

We must first make a brief reference to the price candle data. Each candlestick is consisted of four highest, lowest, opening, and closing prices. The lowest price is formed. Other short-term inputs predict these four prices for five candlesticks of the previous five minutes.

Structure of the first model:

The general prediction model consists of three types of more detailed models, each of which was constructed with the help of deep learning and artificial neural network. The general prediction model is a model based on the majority vote, the output of which is the output that has a majority among the minor models. For example, if at least two minor models vote to increase by more than fifteen points, the output of the overall model will be the same. In general, the partial and general models are as follows:

The first model is a combination of two LSTM (Long short-term memory) deep neural networks. The input of a network is a one-hour long-term input in time series, and the input of another network is the shortterm inputs of five minutes mentioned above, which are also given to the network in time series.

The second model consists of a combination of two deep CNN (convolutional neural network) neural networks, one of which, as above, is five minutes shorttime data and the other is the long-term hourly data.

The third model is a combination of two MLP (multilayer perceptron) neural networks and has the same input as the above models. The general model is also a model based on the majority vote of the above 
three models. The following figure shows the design of the first model:

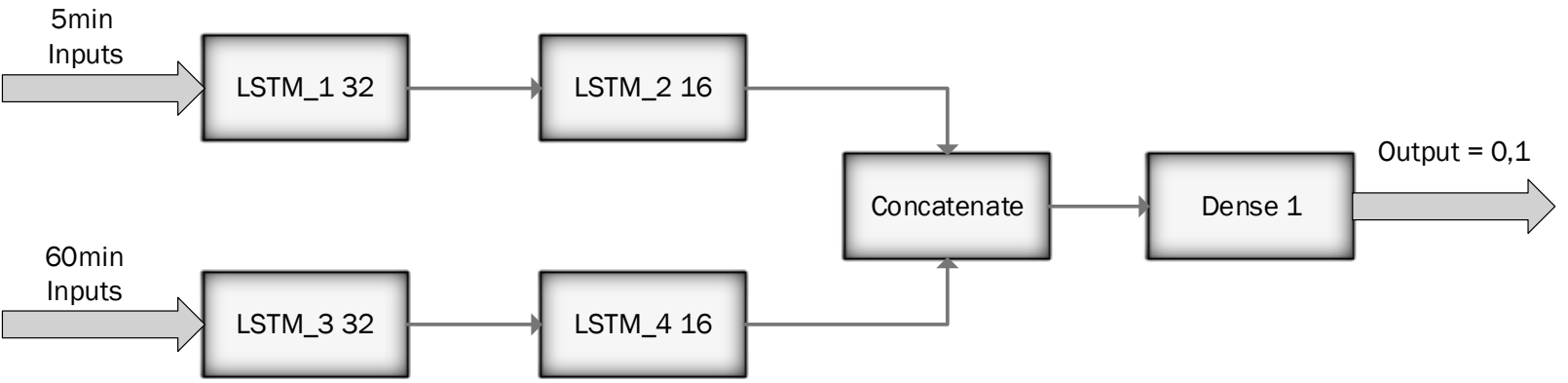

Figure 1. design of the first partial model.

The following figure shows the structure of the second partial model:

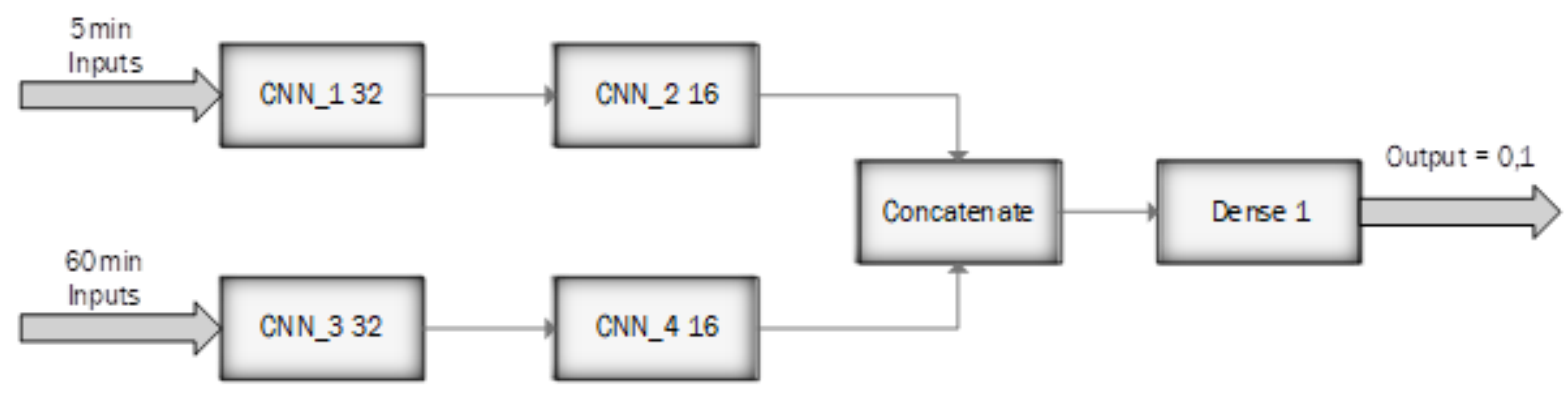

Figure 2. The second partial model structure.

The following figure shows the structure of the third partial model:

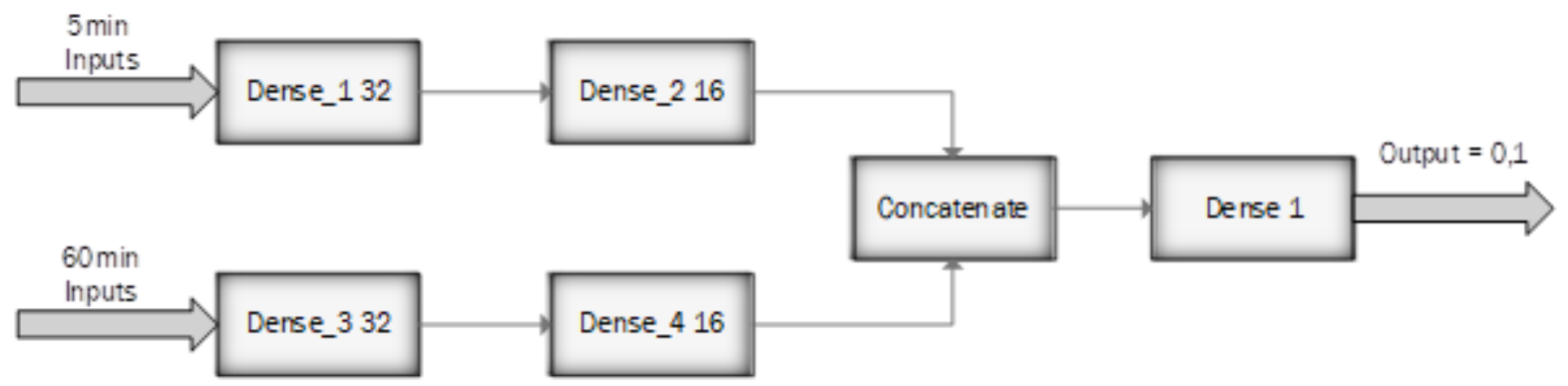

Figure 3. Third partial network structure.

\section{The results of the first partial model}

In this section, we review the results of the first model for the points of the first group. As mentioned, there are 3785 points belonging to this group, which we divided into $70,15,15$ ratios. We used $70 \%$ of them to training the model, then with $15 \%$ of the data that the model has not observed before, we performed the model validation process. In the end, we tested the model for $15 \%$ remaining.

As we said, neural network models have various parameters that we need to adjust according to our problem. After we designed our model on the training data, we simultaneously evaluate our model on the validation data. This approach allows the model to be tested on data which has not observed before. Then we change the model parameters again to create a new model and test it on the validation data to get better results. We continue this process until we can get good results from the validation data. However, excessive frequency of this process leads to the penetration of validation data features on the model and jeopardizes the generality of the model.

To learn the first model, 700 frequencies with a batch size of 32 were used. At the same time, in each iteration, we check the value of the cost function and the accuracy of model on the training and validation data. The following two figures show the trend of changes in accuracy and cost function for training and validation data in each frequency. 


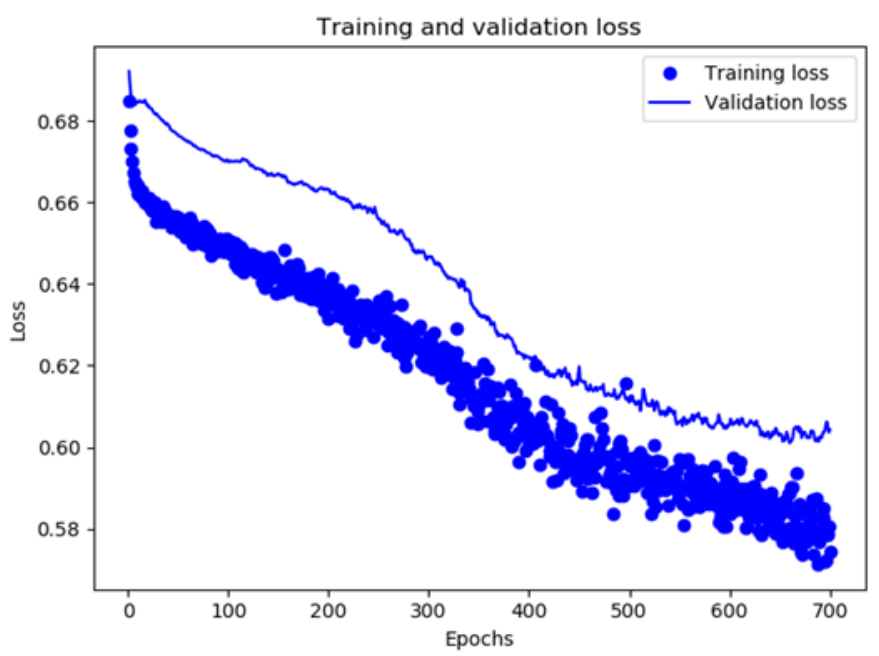

Figure 4. Cost and frequency learning function.

Regarding the above figure, it can be said that the value of the cost function per frequency is decreasing to 0.6043 for the validation data. This model is learned with the parameters mentioned in the third chapter for the first model. To design the first model, various parameters with various designs were studied and finally, by testing them on validation data, the final composition was selected.

As it is observed, the cost reduction slope of the cost function is high first and then this slope decreases, and after a while we will not see such a decrease for the model. It seems that after 700 frequencys in learning the model, the learning process is complete. Now the question that adescent $\mathrm{s}$ is what happens if we increase the number of times of learning, for example to 1500 times? In this case, the model suffers from overfitting and loses its generality. In this case, we will see the cost function increase for the validation data that the model has not observed before.

Now we will examine the process of changes in the accuracy of model and measure it, which is presented in the figure below.

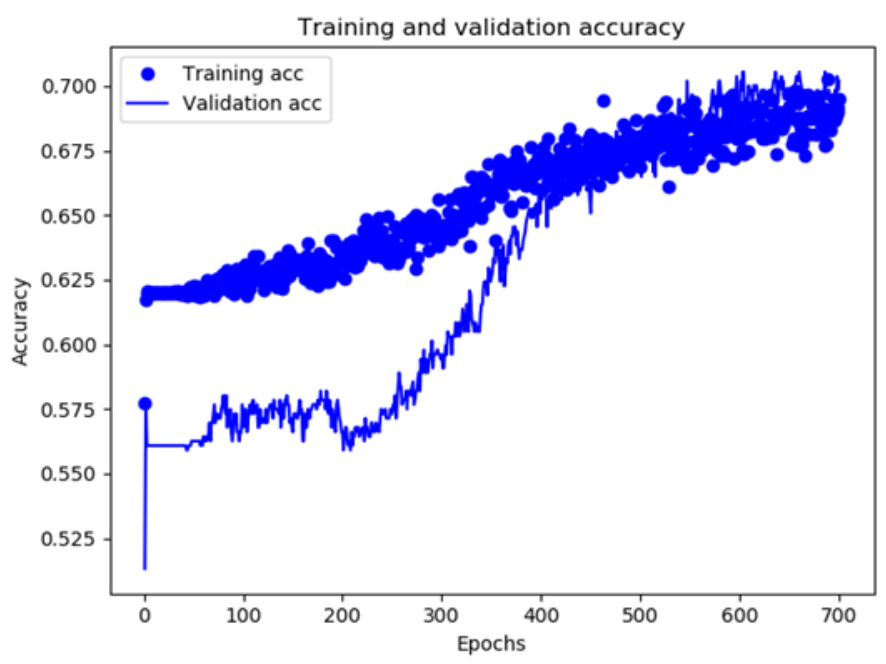

Figure 5. Accuracy and frequency of learning.

As it is observed, the accuracy of the model is increasing with each frequency. At the beginning of the learning path, the increase in the accuracy of the model is high and gradually decreases. The accuracy of the model, as it is observed, reaches about $71 \%$ after 700 rounds of frequency. As the number of learning times increases, as it has been said, we run the risk of overfitting, in which case the accuracy of the model increases for training data but decreases for validation data.

First, we describe the confusion matrix and its components, which are commonly discussed in binary classification problems. As it was said, the output of the model will be two numbers one and zero. 
Prediction one means that the model predicts an ascent of more than 15 points, and the number zero means that the prediction of the ascent model will not be more than 15 points. According to this issue, test data are divided into four groups.

The first group is the data for which the number one model is predicted and in fact the class related to it, is one. This set of positive data is called true positive.

The second group is data for which the model predicts the number zero, but in reality, their label is one. This category is called false negative or false zero.
The third group is the data for which the model predicts the number one, but the label is zero for them. This category is called false one or false positive.

The fourth group is the data for which the model predicts zero and the correct label for them is zero. This category is called true zero or true negative.

Now, by introducing these four sets of confusion matrix, the following figure is drawn.

Table 1

Confusion matrix.

\begin{tabular}{lcc}
\hline & Zero predicted & One predicted \\
\hline True zero & True negative & False Positive \\
True one & False negative & True positive \\
\hline
\end{tabular}

Now it is examined the confusion matrix of the first model for test data and analyze its results.

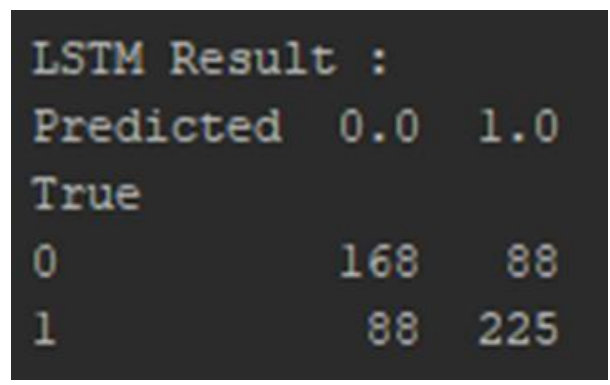

Figure 6. Results of the first model.

As it is observed in the matrix numbers, the number of ascent predictions more than 15 points is 313 times, which 225 ones are correct. This means that if we enter the buying position according to the model forecasts, we have acted correctly with $71.8 \%$ accuracy, which will be an acceptable performance for a trader. The number of points that really increased by more than 15 points is equal to 313 and the number of actual points with label zero is 256 . As we can see, the number of points in the first group is more. If a trader enters a trade with a simple strategy and without always-buy learning of the points of first group, he will have an accuracy of $55 \%$.

This calculation shows two important points: The first point is that the points of the first group, as mentioned, show a good potential for short-term growth, and this is confirmed by strong signals. The second point refers to the strength of the forecasting model, which enables the trader to increase his accuracy by $16.8 \%$.

Now we examine the points for which the model has had predictions other than an increase of more than 15 points. In this case, the model prevents the trader from being in the buying position. The total number of predictions with label zero is 256, which 168 ones are correctly predicted. This forecast allows the model to correctly prevent the trader from losing up to $74 \%$.

Looking at the whole table, we can see that the model correctly identified 393 points out of 569 test points. Therefore, it can be said that the overall accuracy is $69 \%$, which is a good accuracy for a Forex trader.

With examining the accuracy of the model in detail and general, we can understand the strength of the LSTM network and its role in empowering the trader.

\section{Results of the second and third partial models}

We will now briefly review the results of the second and third partial models:

The results of the second partial model for the test data in the form of a confusion matrix are as follows: 


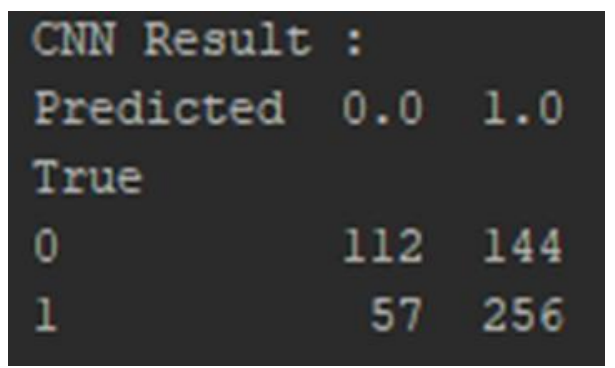

Figure 7. Results of the second model.

As it is observed, in 400 cases the second partial model predicts that we will face a descent of more than 15 points until the next collision of the averages, which has an accuracy of $64 \%$. This model empowers the trader to buy up to $9 \%$ more than a simple strategy, which is a good number for a trader with a short-term strategy.

The model also barred traders from trading 169 times (Prediction Zero), which does so with $66.2 \%$ accuracy. In fact, we have seen the currency pair ratio descent 57 times, with the model preventing the trader from making a profit, which is equivalent to an error about 33.8 ; it is an acceptable rate.
Looking at the data, it can be observed that out of 569 test points, the second model has had 386 correct judgments, which is equivalent to $67.8 \%$ accuracy.

As it is observed, the second partial model, despite its relatively good accuracy, performed worse than the first model, which has LSTM layers. This result was also predictable because the structure of LSTM layers is highly proportional to time series numbers. This layer uses both long-term and short-term memory in its prediction, and gives a special place to the prediction for a closer time. The results of the third partial model in the form of a confusion matrix are as follows:

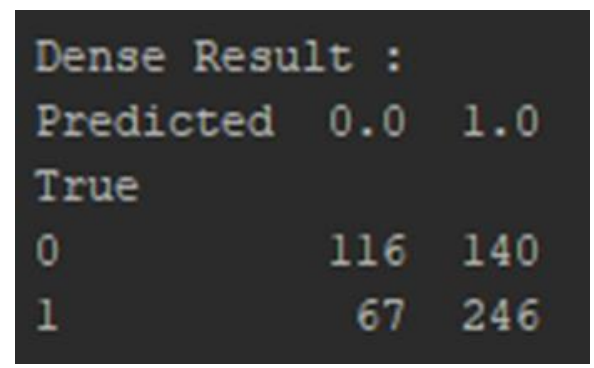

Figure 8. Results of the third model.

As it is observed, in 386 cases the second partial model predicts that we will descent more than 15 points until the next collision of the averages, which has an accuracy of $63.7 \%$. This model empowers the trader by up to $8.7 \%$ compared to the simple always-buy strategy, which is a good number for a trader with a short-term strategy.

The model also barred traders from trading 183 times (Prediction Zero), which does so with 63.8\% accuracy. In fact, it is seen the currency pair descent 67 times, with the model preventing the trader from making a profit, which is equivalent to an error about 36.2; it is an acceptable rate.

Looking at the data, it can be seen that out of 569 test points, the second model has had 362 correct judgments, which is equivalent to $63.6 \%$ accuracy.

As it is observed, the third partial model, despite its relatively good accuracy, performed worse than the first and second models. This result was also very predictable because this network has a simpler structure than previous networks and as a result could not recognize some patterns well. This shows why we need networks with more complex structures to build models.

\section{General model results}

As it has been said, the general model is a majority vote model, the output of which we showed in the above relations. Hybrid models generally produce better results than partial models. Because each partial model has its own approach to events modeling, and the hybrid model uses all of them to predict simultaneously. But in the majority vote model we considered, the hybrid model is less accurate than the first partial models. We will first analyze the results of the hybrid model and then discuss its lower accuracy than the partial models. 


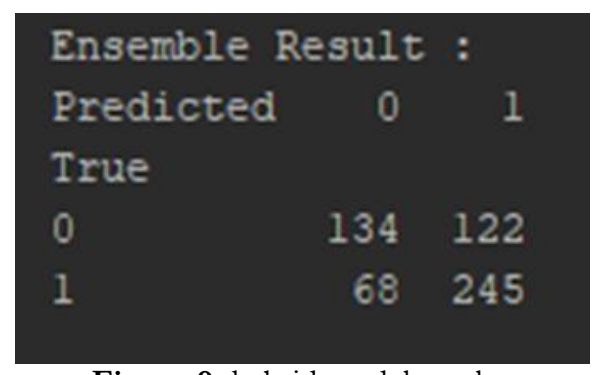

Figure 9. hybrid model results.

As it is observed, in 367 cases the hybrid model predicts that we will descent more than 15 points until the next collision of the averages, which has an accuracy of $70.8 \%$. This model empowers the trader up to $15.8 \%$ compared to the simple always-buy strategy, which is a good number for a trader with a short-term strategy.

The model also barred the trader from trading 202 times (Prediction Zero), which does so with 66.3\% accuracy. In fact, it has been seen 68 times of the currency pair ratio, which has prevented the trader from making a profit, which is equivalent to an error about $33.7 \%$; it is an acceptable rate.

Looking at the data, it can be seen that out of 569 test points, the overall model has made 367 correct judgments, which is equivalent to an accuracy of $64.5 \%$. Now we analyze the results of three models together.
As it is observed in the case of part one, which determines the most important factor in the strength of the algorithm (because this prediction leads to a buy position), the first model is the most accurate, and then the hybrid model is in second place. This event may be due to the relatively high accuracy difference between the first model and the other two models, which combined with other models can lead to the weakness of the overall model. In other words, when the first high-powered model was placed next to the other two weaker models, it could not make a more powerful combination.

In the case of the correct part zero, we get the same as before, but in terms of the overall accuracy, the second model is in second place and the hybrid model is in third place. This event may be due to the weaker third model, that its combination with the above two models can reduce the accuracy of the hybrid model.

Table 2

Comparison of models' results for the points of first group.

\begin{tabular}{|c|c|c|c|}
\hline & Accuracy of prediction one & Accuracy of prediction zero & Total accuracy \\
\hline Model & $\% \vee \varepsilon, \Gamma$ & $\% 70$ & $\% 79$ \\
\hline Second model & $\%$ \%, r & $\%\urcorner \leq, \wedge$ & $\% 70,0$ \\
\hline Third model & $\% 79, \wedge$ & $\% \pi r, \varepsilon$ & $\% 70, \mathrm{~V}$ \\
\hline Hybrid model & $\% \vee \cdot, V$ & $\%$ \% & $\% 7 V, 0$ \\
\hline
\end{tabular}

The results of the models for the points of third group:

Now, it is briefly a review on the results of the models for the points of third group.

Table 3

Comparison of model results for the points of third group.

\begin{tabular}{|c|c|c|c|}
\hline & Accuracy of prediction one & Accuracy of prediction zero & Total accuracy \\
\hline First model & $\% \vee \backslash, \wedge$ & $\% \vee \leq$ & $\% 79$ \\
\hline Second model & $\%$ & $\%$ & $\%$ \% , \\
\hline Third model & $\% \pi r, v$ & $\% \pi r, \Lambda$ & $\%$ \\
\hline Hybrid model & $\% \vee \cdot, \wedge$ & \% & $\%\urcorner \leq, 0$ \\
\hline
\end{tabular}

As it is observed in the case of part one, which determines the most important factor in the strength of the algorithm (because this prediction leads to a buy position), the first model is the most accurate, and then the hybrid model is in second place. Again, this event may be due to the relatively high accuracy difference between the first model and two other models, which combination with other models can weaken the overall 
model. In other words, when the first high-powered model was placed next to the other two weaker models, it could not make a more powerful combination.

In the case of correct zero data, the hybrid model is almost as accurate as the second model and is in third place with a slight difference. In terms of overall accuracy, the hybrid model is in second place with $2 \%$ difference.

\section{Conclusion}

As it is observed, with examining various statistical models that have been used by researchers and traders in the past, we have come to the conclusion that neural network-based models are models with high predictive accuracy. Among these models, deep models showed more modeling power. Therefore, we based our model on deep neural networks with various layers, and finally presented a hybrid model with several inputs as well as a majority vote model.

We used a scalping strategy to determine the data needed to build and test the model. With the help of this strategy, we obtained important points and divided them into two categories. Statistical analysis of these two categories showed that these points have a high potential for ascending or descending, and paying attention to them can make a profit for the trader.

With the help of these points, three minor models were made and then tested. Comparing the results, it was found that the first partial model, which includes LSTM layers, had the highest prediction accuracy. The first partial model for the first group increases the success rate of the scalping strategy for the trader to $71.8 \%$. In the case of the third group, this model also raises the success rate of the scalping strategy for the trader to $74 \%$.

As a result, we saw that building a hybrid model with deep LSTM layers and with multiple inputs based on the scalping strategy, we were able to increase the success rate by more than $70 \%$. This rate of power of LSTM deep neural networks in predicting shows the direction and magnitude of market movement in both uptrend and downtrends and shows that these networks are very suitable for time series data. We also saw that the strength of LSTM layers was greater than torsional layers and multilayer perceptron's, and even the overall hybrid model.

\section{References}

1. Zhang G, Eddy Patuwo B, Hu MY. Forecasting with artificial neural networks: The state of the art. Int $J$ Forecast. 1998; 14(1): 35-62.

2. Lawrence R. Using neural networks to forecast stock market prices. $1997 ; 1-21$.

3. Tsibouris G, Zeidenberg M. Testing the efficient markets hypothesis with gradient descent algorithms. Neural Net Cap Market. 1995; 127-136.

4. Tsai CF, Hsiao YC. Combining multiple feature selection methods for stock prediction: Union, intersection, and multi-intersection approaches. Decis Support Syst. 2010; 50(1): 258-269.

5. Hadavandi E, Shavandi H, Ghanbari A. Integration of genetic fuzzy systems and artificial neural networks for stock price forecasting. Knowl Base Syst. 2010; 23(8): 800-808.

6. Barak S, Arjmand A, Ortobelli S. Fusion of multiple diverse predictors in stock market. Inf Fusion. 2017; 36: 90-102.

7. Ballings M, Van Den Poel D, Hespeels N, Gryp R. Expert systems with applications evaluating multiple classifiers for stock price direction prediction. Expert Syst Appl. 2015; May.

8. Sowmya R, Suneetha KR. Data mining with big data. Proccidings of the $11^{\text {th }}$ International Conference Intelligent Systems Control. ISCO 2017; 26(1): 246250.

9. Tsai C-F, Wang S-P. Stock price forecasting by hybrid machine learning. Proccidings of the International Multiconference Engineering and Computer Science. 2009; I: 2210.

10. Rather AM, Agarwal A, Sastry VN. Recurrent neural network and a hybrid model for prediction of stock returns. Expert Syst Appl. 2015; 42(6): 3234-3241.

11. Norris E. Scalping: small quick profits can add up. Investopedia. 2019;

https://www.investopedia.com/articles/trading/05/sc alping.asp

\section{SJAMAO}

Copyright: (C) 2020 The Author(s); This is an open-access article distributed under the terms of the Creative Commons Attribution License (http://creativecommons.org/licenses/by/4.0), which permits unrestricted use, distribution, and reproduction in any medium, provided the original work is properly cited.

Citation: Sabbaghi Lalimi AH, Damavandi H. Using Machine Learning Methods in the Financial Market for Technical Analysis Based on Hybrid Models. SJAMAO, 2020; 2(4): 1-11.

https://doi.org/10.47176/sjamao.2.4.1 